\title{
Editorial
}

\section{NEW VIEWS ON CELL DEATH}

\author{
Marian Adamkov \\ Department of Histology, Jessenius Faculty of Medicine in Martin, Comenius University in \\ Bratislava, Martin, Slovakia
}

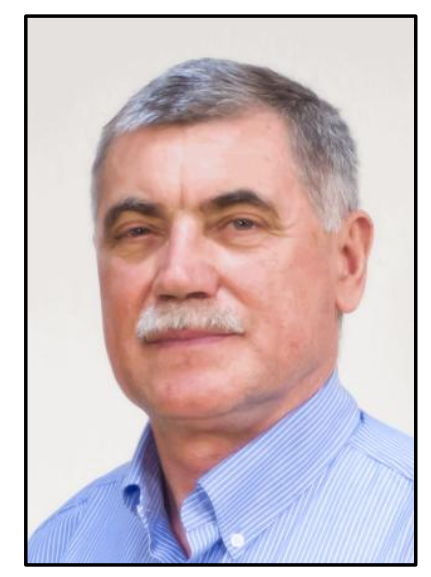

Cell death introduces an important phenomenon in normal organ histogenesis, in continuous kinetics of healthy tissues, in pathogenesis of injury and diseased states, and also in the treatment of a broad spectrum of diseases. The study and understanding of cell death is one of the foundations in human pathobiology and in therapy of diseases. Cell proliferation and cell death feature important mechanisms which maintain normal cellular homeostasis of tissues.

However, there is a number of ways how cells can die. Cell death is principally classified into three major categories which are the most widely known:

- apoptosis (Type I)

- autophagy (Type II)

- necrosis (Type III)

Regulated removal of selected cells by apoptosis differs from lytic destruction of cells by necrosis. Autophagy is, on the other hand, an intracellular catabolic process which occurs by lysosomal degradation of damaged organelles. However, it is recognised that the morphological image of each individual type of cell death may partly overlap. Nowadays therefore it is also accepted that cell death can represent one continuous process, with necrosis at one end of the morphological spectrum and apoptosis at the other end. Through the development of the knowledge base, molecular mechanisms of individual forms of cell death are gradually becoming understood. This can shed light on further uncertainties resulting from the morphological image.

Until recently the various types of cell death have been defined, based mainly on morphological criteria whereby no molecular and biochemical aspects were taken into account. The international group NCCD (The Nomenclature Committee on Cell Death) presents new views on the processes relating to cell death. The NCCD describes in detail the molecular background of the particular types of cell death and suggests new terminology which is based on the most recent findings in this area. For example, it is suggested that nomenclature such as "the percentage of apoptotic cells", "the percentage of necrotic cells", "the percentage of dead cells" and "the percentage of surviving cells" should be replaced with more accurate and concise terms. The use of new terminology for those processes that relate to cell death is recommended, for example "the percentage of chromatin condensed cells", "the percentage of cells with DNA fragmentation", "the percentage of cells with low transmembrane potential of the mitochondria", "the percentage of PI-positive cells" (Propidium lodide), "the percentage of cells positive for Caspase-3 and "the percentage of TUNEL (IdT-mediated duTTP-biotin nick end labelling, TUNEL assay)-positive cells". The NCCD also proposes to change the term "the percentage of autophagic cells" with a new term "the percentage of vacuolated cells", etc. The NCCD further recommends diagnosing cell death by applying more than one method in order to 
prevent the erroneous assessment of possible artefacts.

Enormous scientific development continually brings new knowledge in all aspects of cell death study. Therefore the NCCD proposes the precise definition of all types of cell death on molecular, biochemical, pharmacological and functional bases. Morphological manifestations of cell death from this perspective are not considered to be essential.

The process of cell death is reversible until the first irreversible phase; the so called "point-of-noreturn"; is reached. This step could be represented by the significant activation of caspases, by the loss of mitochondrial transmembrane potential, by the complete mitochondrial outer membrane permeabilization and by the phosphatidylserine on the surface of the cell. According to the NCCD, in the absence of distinct biochemical criteria for the irreversible phase, a cell can be considered to be dead when at least one of the following molecular or morphological characteristics is present:

- the loss of cell membrane integrity which is demonstrated by the incorporation of vital dyes, e.g. $\mathrm{PI}$ in vitro,

- the cell, including the nucleus, displays a distinct fragmentation in apoptotic bodies,

- the phagocytosis of apoptotic cells / fragments by surrounding cells in vivo.

However, it is essential to differentiate "the dead cells" from "the dying cells" which may arise in a wide spectrum of various biochemical processes, e.g. senescent cells. These are regarded as cellular elements that are still living.

Apart from the three major types of cell death highlighted above, there are additional forms which are more often discussed in literature such as:

- Cornification (Keratinization)

- Mitotic catastrophe

- Anoikis

- Excitotoxicity
- Wallerian degeneration

- Paraptosis

- Pyroptosis

- Pyronecrosis

- Entosis

- MPT - regulated necrosis

- Necroptosis

- Ferroptosis

- Parthanatosis

- NETosis cell death

- Lysosome-dependent cell death

- Immunogenic cell death.

However, apoptosis clearly attracts the most attention by research teams. Apoptosis is a natural process which is essential for the normal structure of all tissues as well as physiological functions. Life in a multicellular organism could not exist without cell death which literally removes any damaged or depleted cells in an ingenious way. Furthermore, there are cells eliminated by apoptosis which have already fulfilled their specific role in the organism; as well as those cells which have only a limited ability to withstand various forms of stress. Apoptosis is aptly described as a programmed / regulated cell death since strictly controlled and programmed intracellular mechanisms literally drive the cell to suicide. By personification of this unique action in the micro-world of an individual cell, it can be concluded that the cell will realise its transient existence in the stark reality of life. It clearly recognises that it has become a surplus requirement in a particular tissue and this leads it to a regulated departure from the conglomerate of surrounding active cells.

Abnormalities of regulatory mechanisms of the apoptotic process are observed in the majority of diseased states. Therefore it is logical that apoptosis is the subject of great interest in modern medicine. Comprehensive understanding of apoptotic aspects in individual diseases can explain their detailed pathogenesis and also consequently influence the targeted therapy. 